is demonstrated; the loss of definition of the bone graft should be noted. Fig. 4 shows the completed radius.

Case II (Fig. 5) was a case of tuberculous disease of the first phalanx of the thumb. Fig. 6 shows the bone graft in the periosteal sheath of the excised phalanx; it should be noted that the articular cartilages have not been interfered with. Fig. 7 shows bone growth round the graft, which is losing density in consequence of absorption. In Fig. 8 we liave the completed phalanx.

Case III was a whitlow of the thumb with necrosis of the phalanges. In Fig. 9 there may be observed the enormous swelling of the soft parts owing to virulent suppuration and poulticing. Fig. 10 shows the bone graft, equal in length to the excised phalanges in situ. In Fig. 11 there should be noted the vacuolation of the bonegraft and segmental reformation of the two phalanges (two months after operation); suppuration has greatly diminished and the soft parts are acquiring a normal outline; there is good movement at the metacarpo-phalangeal joint. Fig. 12 three months after operation, shows disintegration of the bone-graft more marked; the thumb is almost normal in appearance, the sinuses have healed up, and there is improved movement at the metacarpo-phalangeal joint This case is still under observation.

Case IV was a myeloma of the radius; in Fig. 13 the osseous outline of the growth may be noted. In Fig. 14 the tumour is scruped out and the transplant placed in the cavity; there is an "inlay" contact with the healthy end of the shaft. In Fig. 15 invagination of the bone graft by bone growth from the shaft is demonstrated; ossifying lamellae in the "egg-shell" cavity should be noted. In Fig. 16 is shown commencing canalization of the reformed shaft; it should be observed that bone of normal density occupies the space originally filled by the growth.

I am satisfied that the new bone and cellular life generally which result from the stimulating effect of the trans plant are capable of overcoming pyogenic and tuberculous infections (Cases I, II, III) and of reviving normal osteogenesis in bones affected by neoplasmic formations of low imalignancy (Case Iv).

My thanks are due to Dr. Woodburn Morison of Man. chester and Dr. Brice of Dukinfield for the radiograms.

\section{GENERAL ANAESTHESIA AND THE} ATMOSPHERE IN THE OPERATION THEATRE. BY

\section{J. ROSS MACKENZIE, M.D.}

ASSISTANT ANAESTHETIST, ROYAL INFIRMARY, AND TO THE ROTAL HOBPITAL FOR SJGK CEILDREN, $\triangle$ BERDEEN : AND

G. H. COLT, F.R.C.S., ASSISTANT SURGEON, ABERDEEN ROYAL INFIRMART.

IN $1908^{1}$ a note was published on the administration of ether by the open method in which the possibility of using a valved container was described, the valves being placed between the container and the face-piece. This was in the early days of "open" ether. Since that time the adoption of Hewitt's principle of using wide-bore tubes, which tends to abolish stimulation of the respiratory effort and so to lessen stridor and cyanosis, and the provision of a larger evaporating surface for the ether, have given us various pieces of apparatus for the same purpose. One of the best known of these is the Anson and Caldwell-Smith inhaler. Such an apparatus produces as good an anaesthesia as is obtained by the true open ether method, and at the same time considerably lessens the loss of the drug due to direct evaporation into the atmosphere. In any such piece of apparatus at first about 50 per cent., and, when anaesthetic equilibrium is estab. lished, 100 per cent. of the ether used is discharged into the theatre. In fact, at the present time it is not unusual for those engaged in operative work to be surrounded by unpleasant and deleterious vapours which would neither be tolerated nor permitted by law to exist in any ordinary factory. The chief sufferer is the anaesthetist, and next to him the surgeon and those in greater or less proximity to the patient's head. Some persons are more readily affected than others. As later results gastritis and anaemia are not unknown. In field work and in some cases where small rooms are used the effect on the staff is serious. This is notably the case when a flame, a glowing fire, or an electric heater is exposed to the vapour of chloroform, which is transformed into phosgene gas, and is one of the main reasons why operation theatres are heated by steam or water piping. In small and repeated doses this gas produces anaemia. In larger doses it leads to a feeling of tightness and constriction in the chest, most marked about twelve hours after the exposure, and followed by bronchitis of a varying degree of severity.

The "open" ether method, especially when combined with the use of narcotic and other drugs, is very safe, a fact which has undoubtediy had great weight in causing its almost universal adoption as an anaesthetic of choice for the great majority of operations.

We have thought for some years that it ought to be possible to generate the vapours outside the theatre entirely, to deliver them to the face-piece through a wide tube and to remove them by a similar channel, so that the atmosphere of the theatre need never be materially con. taminated. One of us (G. H. C.) mentioned the point to an anaesthetist in 1907, but the matter did not materialize. We are not at present proposing to experiment in this way on any large scale, but we wish in this preliminary note to mention the chief points which seem to us important and which will undoubtedly require consideration in theatre construction in the future, as our trials demonstrate. In this way we hope to interest others who are conversant with the varied conditions existing in different parts of the country. It may be noted that the principle of removing the source of anaesthesia to a distance has been put into practice for some years past in cases in which a preliminary laryngotomy or tracheotomy is performed and the tube connected to a distant "tambour."

At the outset there will be a difference in plan according as to whether the operation is to be performed in an operation theatre or elsewhere. In the case of a theatre a permanent plant could be established just as illuminating gas is laid on. From the generator the tubes would be brought through the floor near the table, with taps, unions, and flexible connexions there, so that the apparatus suitable to the case could be connected to the main supply and exhaust. In some cases a single generating station miglit be arranged to supply to and exhaust from several tlieatres. But when the operation is to be done in a private house a portable apparatus would have to be employed and adapted on arrival to the local conditions. This could be made a simple thing, as we propose to sliow.

In the second place, the source of ether vapour should not allow any loss to occur to the outside air, partly on the ground of expense and partly to aroid contaminating the atmosphere near the theatre, bearing in mind the everpresent risk of explosion from a spark from an electric motor or other cause. To ensure this it would be necessary to generate the vapour at some distance from the air entrance, and perhaps to put a valve stop and fine wire gauze mesh behind it. The strength of the vapour could be regulated by a tap or throttle or other contrivance controlled from the operation table.

Thirdly, there should be a heater for the vapour and a quick-movement thermometer in the face-piece to show the temperature at which the anaesthetic vapours reach the patient. The face-piece itself would comprise an inlet and an outlet valve, but these might be provided for else. where and at a distance if found more convenient and if the $\mathrm{CO}_{2}$ content of the system were not too great. In case any friction should arise in the system it will be necessary to maintain an exhaust at the exit of the fairway of such a degree that the valves would act with the minimum of differential pressure, and so that there would be hardly any deviation from atmospheric pressure inside the face-piece.

Fourthly, it will be necessary to arrange for the delivery to the fairway of nitrous oxide, ethyl chloride, chloroform, oxygen, or other agent as required, and the apparatus should preferably be adaptable to intratracheal methods.

Lastly, the expired vapours should be trapped in a suit. able solvent from which the chief ingredients could be recovered by distillation or otherwise.

It seems to us that this proposal is the ideal that will have to be taken into account in the future in the construc. tion of any operation theatre, and that its adaptation to existing theatres will only be a matter of time. There is little doubt that the initial outlay would soon be wiped out by the saving of the cost of the drugs recovered. 
The present system of rentilation in theatres is often imperfect, neither does it deal with the source of origin of the trouble.

In order to test some of the principles involved in our proposal we have carried out the following trials :

(a) Messrs. Down Bros. constructed for us the apparatus slown in Fig. 1. The point was whether it would be possible to collect the major portion of the ether by maintaining an extraction current of air close to the patient's mouth. The two cups aro stream-lined and lie on each side of the mouth under the gauze used for open ether. The amount of ether vapour caugist in this way appears to be negligible, as judged by the smell.

(i) A coil like a gas-ring was placed under the gauze. The result was better.

(c) Fig. 2 is an illustration of a rough and ready apparatus made from tin and gas-piping. Around the rim, which fits the face, and which consists of a wider tube outside and a narrower one inside, there are two series of holes. One series opens outside the face-piece but inside the "cone," and one opens inside the face-piece. By plentifully covering the cone with wool the smell of ether was lessened, and by adding a small extraction gas-ring (Fig. 3) at the top and covering the top of the cone with sheet rubber, except for the contral hole through which ether was dropped, the smell was still further reduced.

(d) Messrs. Down Bros. lent us their experimental model of the Anson and Caldwell-Smith inhaler, and to the exit valve we attached our suction apparatus. Unfortunately the combination proved unwieldy, but we were able to note that the chief loss of ether was from the vaporizing bag, and that it was steady and continuous, so that clearly the solution of the difficulty lay in hav:n $r$ a closed system with valves. We were not able to command the use of a Kielly's apparatus, and in Rutherford's the bore of the tubes was too narrow for our purpose. Messrs. Down Bros. therefore made ns an inhaler like Silk's, but with. ont the shelf, and fitted it with inlet and outlet valves.

(e) The inlet valre of this apparatus was connected, as shown in Fig. 4, to an ether supply by means of a light flexible tube made of coiled aluminium wire and colotomy tubing. The exit valre was similarly con. nected to an ordinary rubber gas big. The other end of this was led off by nar. rower tubing to a wash bottle containing alcohol, and $a \cdot$ steady stream of air was kept up through this by means of a filter pump. It will be seen that this is a simple system, fulfilling the essential conditions for open ether, but the system

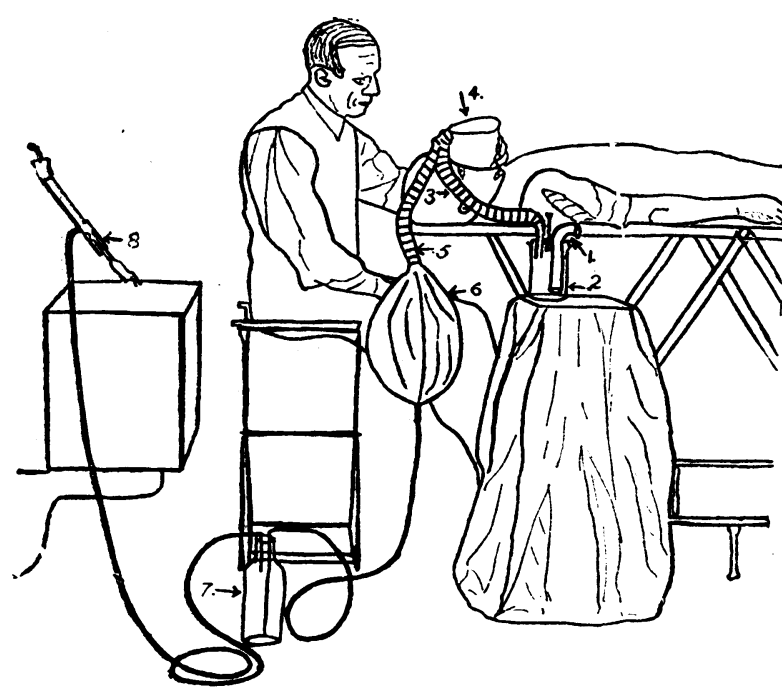

FIci. 4.-1, Air inlet: 2, ether supply: 3, flexible connexion to luask: 4. mask: 5, flexible connexion to bay: 6 , equalizing bag: 7 , wasli-bottle; 8, filter pump with ta: union.
While anaesthesia was maintained there was not the least odour of ether in the neighbourhood of the patient. It was noted that the respirations were not so laboured as is sometimes the case with ordinary open ether, the degree of muscular relaxation was better, and the fatisne of operating, which in many cases seems to be due to the inhalation of ether, was notably absent. Fatigue in operatiug depends on several factors, and at least one-third, if not more, of the ease and facility of an opera. tion depends on the success of the anaesthetic procedure.

The clief point about the use of the wash-bottle is that it traps the ether rapour and prevents it from being sent down the drain, where it is a source of danger. The alcohol evapo. rates also, and a bettcr system would be to have a second wash-bottle of water in the exhaust circuit. In actual practice the total amount of ether let down the sink during one operation would probably not be dangerous, so that for use in a house it would not be necessary to employ a wash. bottle, but if it were it should be a single one, small and made of metal. The use of a wash-bottle necessitates a slightly stronger exhaust. Alterna. tively, by leading the filter pump exhaust ontside the house, the use of the wash-bottle becomes unnccessary.

For use in private, or as a tem. porary measure in a theatre, we conceive that a relatively short but wide ether bottle would be led off to the right-hand side, or alternatively the chin end of the mask. The mask woald be reduced in height as much as possible, as it is notable that the unwieldiness of anaesthetic apparatus depends directly on its lieight, and its capacity should be minimal to aroid the $\mathrm{CO}_{2}$ factor. In preference it should contain a Ticehurst system ${ }^{2}$ for the self-warming of the ether vapour, itself an enormous advance on current practice and the expired air should leave through the left-hand side, or alternatirely the forehead end, of the mask direct through an exit ralre to the bag. The other end of the bag should be led off by tubing to a filter pump operated from the ncarest sink or garden tap. Con. venient tap unions are in common use for liose pipes, and this method lias given us satisfaction provided a safety tape is attached from the union to the tap. We think that the filter pump should be made of metal. In permanent installations, and in cases where there are suitable conveniences, a motor driven air pump will replace the more simple arrangement.

The only serious diffi. culty that we foresee others will have is in providing for the use of an artificial airway. It might be possible to arrange a window in the lower portion of the mask and to make the join a sliding fit with a bead edging of leather to accommodate one of the patterns of airway in use.

The whole question is important at the present day and offers some degree of scope to those interested. There can be little doubt that a successful system would be a great boon to the anaesthetist, surgeon, and theatre staft generally. We are at present engaged on the details of a small portable apparatus.

IRTERENCES

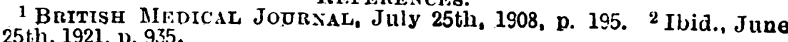
inlet pipe.

'The trials with this system were very satisfactory. 